\title{
Self-Assembly of Silver Metal Clusters of Small Atomicity on Cyclic Peptide Nanotubes
}

\author{
Miguel Cuerva $^{\# \S}$, Rebeca García-Fandiño ${ }^{\dagger}$, Carlos Vázquez-Vázquez ${ }^{\#}$, M. Arturo López-Quintela ${ }^{\#}$ Javier \\ Montenegro $^{\dagger *}$ \& Juan R. Granja ${ }^{\dagger *}$ \\ $\dagger$ Center for Research in Biological Chemistry and Molecular Materials (CIQUS), Organic Chemistry Department, University of Santiago de \\ Compostela (USC), Santiago de Compostela, Spain. ${ }^{\# T e c h n o l o g i c a l ~ R e s e a r c h ~ I n s t i t u t e ~(I I T), ~ P h y s i c a l ~ C h e m i s t r y ~ D e p a r t m e n t, ~ U n i v e r s i t y ~ o f ~}$ \\ Santiago de Compostela (USC), Santiago de Compostela, Spain. *e-mail: javier.montenegro@usc.es; juanr.granja@usc.es.
}

\begin{abstract}
Subnanometric noble metal clusters, composed by only a few atoms, behave like molecular entities and display magnetic, luminescent and catalytic activities. However, non-covalent interactions of molecular metal clusters, lacking of any ligand or surfactant, have not been seen at work. Theoretically attractive and experimentally discernable, van der Waals forces and non-covalent interactions at the metal/organic interfaces will be crucial to understand and develop the next generation of hybrid nanomaterials. Here, we present experimental and theoretical evidence of non-covalent interactions between subnanometric metal (0) silver clusters and aromatic rings and their application in the preparation of 1D self-assembled hybrid architectures with ditopic peptide nanotubes. Atomic force microscopy, fluorescence experiments, circular dichroism and computational simulations verified the occurrence of these interactions in the clean and mild formation of a novel peptide nanotube and metal cluster hybrid materials. The findings reported here confirmed the sensitivity of silver metal clusters of small atomicity towards non-covalent interactions, a concept that could find multiple applications in nanotechnology. We conclude that induced supramolecular forces are optimal candidates for the precise spatial positioning and properties modulation of molecular metal clusters. The reported results herein outline and generalize the possibilities that non-covalent interactions will have in this emerging field.
\end{abstract}

Keyworks: cyclic peptide. nanotubes. metal cluster. self-assembling.

The emergence of molecular-like properties in subnanometric metal (0) clusters (SNMCs) has enable new opportunities in chemistry, nanotechnology and materials science..$^{1-5}$ The energy level quantization in metal clusters of small atomicity triggers the emergence of promising luminescent, magnetic and catalytic properties. ${ }^{3,4,6-10}$ The recent advances in surface passivation and templated synthesis (e.g. mercapto derivatives, DNA, polymers, polyelectrolytes, proteins, dendrimers, etc.) allow the access to metal clusters with controlled size. ${ }^{4,6}$ Furthermore, separation techniques such as liquid extraction, polyacrylamide gel electrophoresis, and high pressure liquid chromatography allow the purification and isolation of metal clusters. ${ }^{4,6}$ In this respect, the recently secured synthetic access to pure SNMCs clearly demands innovative tools to enable their precise manipulation at the nanoscale $8,11,12$ in the development of novel composites, hybrid catalytic systems and nanoelectronics. Unfortunately, the current protocols for nanoparticle generation and/or controlled deposition suffer from drawbacks associated with the precision in surface coverage, the heterogeneity of the resulting nanoparticles and/or the sensitivity of materials to the required tedious or harsh experimental conditions. ${ }^{13-15}$ Hypothetical non-covalent interactions of SNMCs represent an unexplored, clean and innovative opportunity for the adjustment of the spatial position and the molecular properties of these metal clusters. Furthermore, using supramolecular interactions for the self-assembly of neat metal clusters would overcome the current limitations of synthetic protocols such as the presence of undesired surfactants, salt buffers and chemical reagents (e.g. $\mathrm{NaBH}_{4}$ ) required for the generation of molecular metal clusters. ${ }^{1,3,5,16}$

In the recent years many techniques have been developed in order to achieve the arrangement of nanoparticles over different templates or surfaces. ${ }^{3,6,8,17}$ Suspended single wall carbon nanotubes have been used as templates for the vapor deposition of a wide range of metal nanoparticles ( $\mathrm{Pd}, \mathrm{Au}, \mathrm{Fe}, \mathrm{Al}, \mathrm{Pb}){ }^{3,6}$ This methodology was applied in order to overcome the weak interaction of the metal nanoparticles with the $\pi$ surface of the carbon nanotubes. ${ }^{1,5,6,18-21}$ In other examples, the hydrophobic interactions between the surfactants that are generally employed for the dispersion or stabilization of nanostructures, have been used to place large gold nanoparticles over fullerene or carbon nanotubes. ${ }^{8,10,12,22}$ Small nanoparticles have been nucleated (or synthesized) from cationic gold $\left(\mathrm{Au}^{+}\right)$over carbon nanostructures. ${ }^{13,23}$ The same type of templated salt reduction has been described for the size selective preparation of gold nanoparticles in the presence of cyclodextrins templates. ${ }^{16,24}$ The adsorption of ligand-coordinated metal clusters into zeolite pores followed by thermal ligand removal has been elegantly applied for the synthesis of neat metal clusters. ${ }^{17,23,25}$ Corma et al have recently reported the interesting catalytic properties of tricomponent systems made of polyallylamine surfactants, multiwall carbon nanotubes (MWCNTs) and subnanometric gold (0) clusters, although they did not go into details about the interactions of the metal clusters with the surfactant-stabilized MWCNTs. ${ }^{3,24,26}$ The interaction of cationic silver $\left(\mathrm{Ag}^{+}\right)$with anionic DNA has also been employed for the size selective synthesis of silver clusters by the in situ reduction of the corresponding ionic salts. . $^{1,518-21,27-29}$ In these reported synthetic protocols, the macromolecular hosts (e.g. DNA, cyclodextrins, zeolites, etc.) control the size of the clusters guests by inhibiting the unrestrained nucleation of metallic nanoparticles. However, in the approach reported here, the supramolecular host adapts its structure to direct the 1D self-assembly of the cluster guest, which size is previously fixed by synthetic and purification protocols. ${ }^{10,22,28-31}$ 
a)

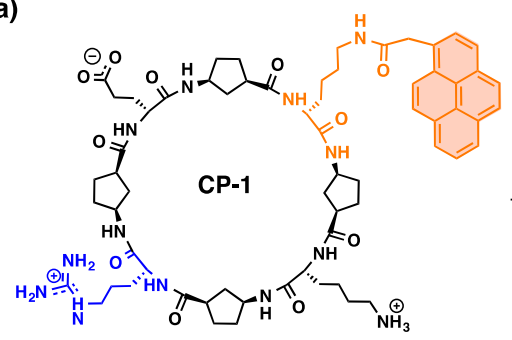

b)

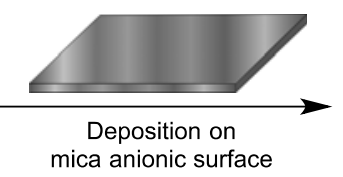

e) -2.0

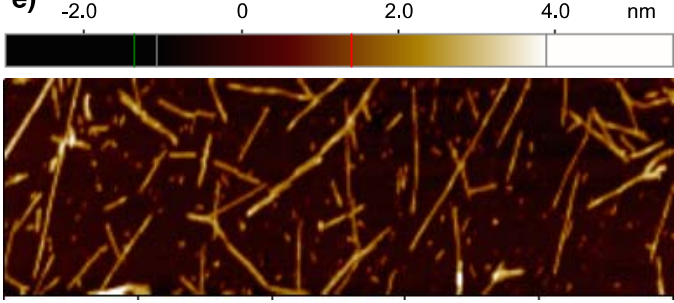

f)
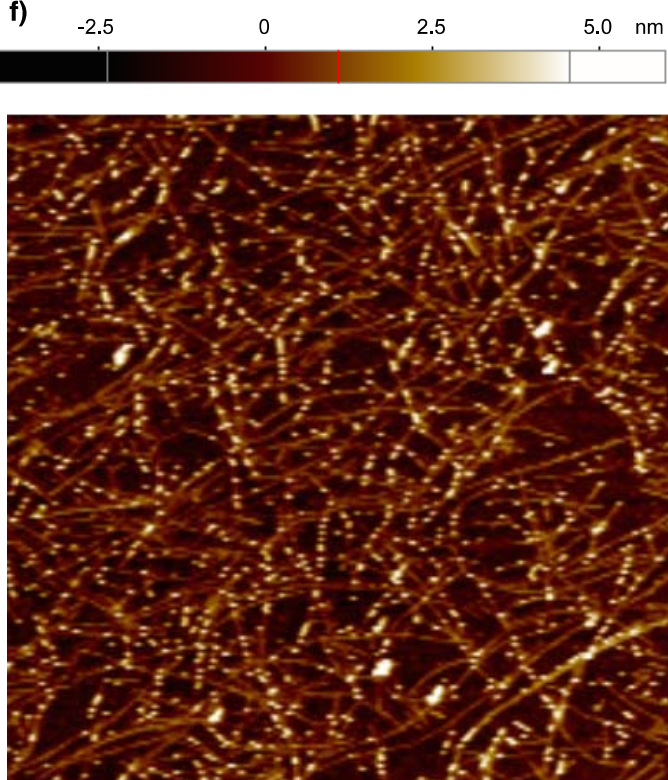

c)
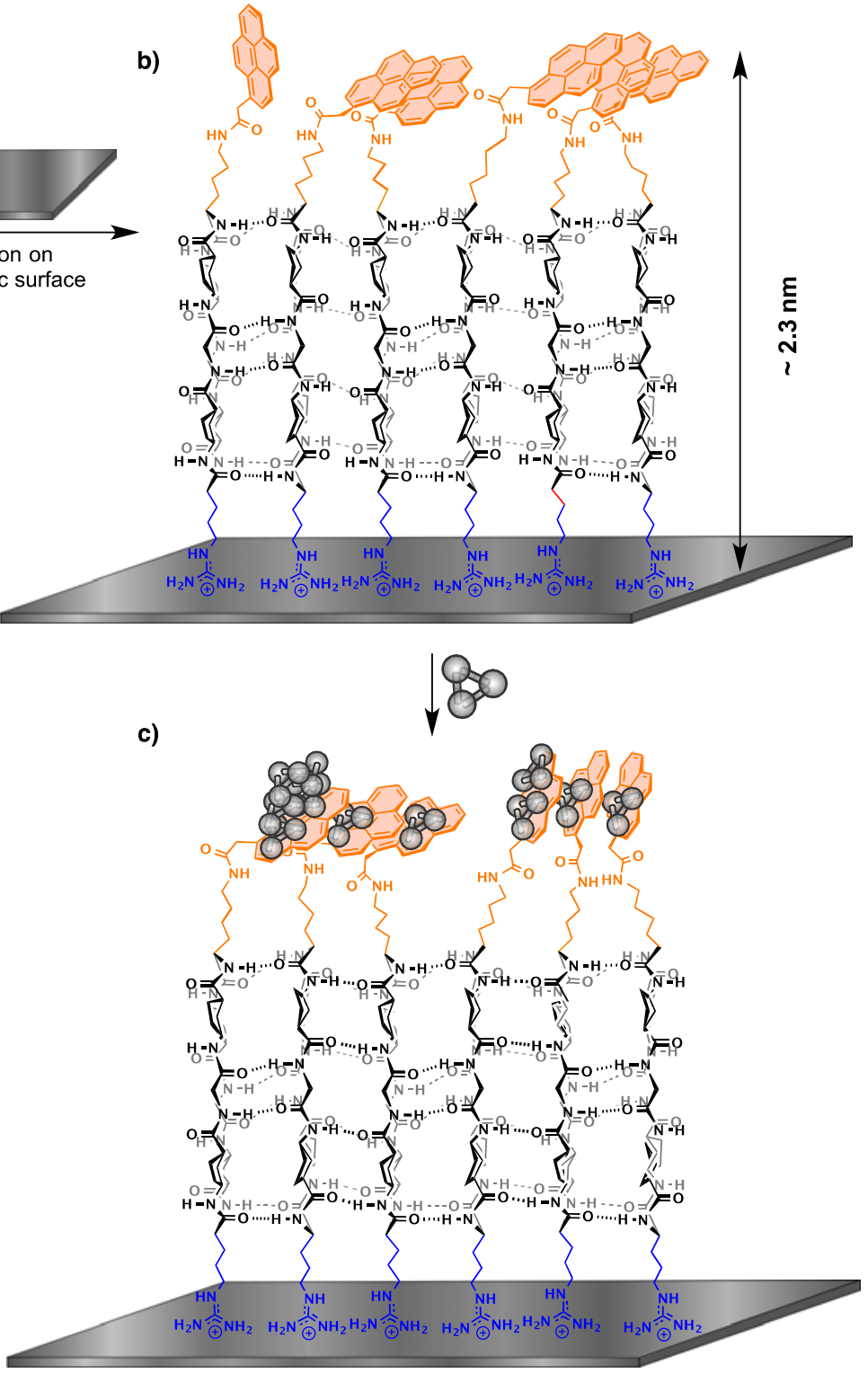

Figure 1. Proposed model for the coupling between SCPNs and Ag 3 clusters and general AFM images for hybrid model structures. a) Structure of CP-1. b) Expected model for the attachment of an SCPN to an anionic surface. The cationic arginine (pH $\sim 2.5)$ interacts with the mica and the hydrophobic interactions of the pyrene residues enhance nanotube self-assembly. c) Incubation of nanotubular arrays with silver clusters aqueous solutions (enriched in $\left.\mathrm{Ag}_{3}\right)$ results in spontaneous cluster deposition on SCPNs $\left(0.8 \times 2.5 \mu \mathrm{m}^{2}\right)$. e) General AFM topography micrographs of pure nanotubes with average heights of $\sim 2.3 \mathrm{~nm}\left(1.65 \times 5 \mu \mathrm{m}^{2}\right)$. f) Metal cluster alignment showing punctuated increases of heights (between $\left.0.5-1 \mathrm{~nm}\right)$ depicting cluster self-assembly and aggregation on peptide nanotubes $\left(5 \times 5 \mu \mathrm{m}^{2}\right)$.

Discrete clusters lacking of any ligand or surfactant are therefore different from charged metal salts, surfactant or ligand-stabilized metal clusters and larger metal nanoparticles. SNMCs behave like genuine molecules in terms of magnetism, catalysis and luminescence and they might also establish non-covalent interactions with other molecular entities by means of dipole-induced forces. In effect, it has been shown that small copper clusters of this type (3-14 atoms) can exhibit strong solvatochromism (absorbance/emission) depending on the solvent polarity. ${ }^{23,29,30}$ Additionally, it has recently been computed that cluster- $\pi$ interactions play important roles in the interactions of twelve atoms silver and gold clusters with the aromatic clouds of nucleobases. ${ }^{24,32}$ Although covalent bonds with functional groups (thiol, phosphorous) are possible depending on the cluster size/structure, it is also reasonable to postulate the existence of dynamic dipole (or quadropole) induction for the ground and excited states of single SNMCs. However, and apart from these encouraging experimental ${ }^{23,25,29}$ and computational results ${ }^{24,26,29}$ non-covalent interactions (van der Waals forces and/or $\pi$-stacking and hydrophobic effects...) have not yet been considered and applied as experimental tools to influence and manipulate subnanometric metal $(0)$ clusters at the molecular level.

We here report that uncharged molecular-like SNMCs, smaller than $10 \AA$, can undergo genuine non-covalent interactions with other supramolecular entities. These interactions have been applied to prepare self-assembled hybrid architectures of neat silver clusters $\left(\mathrm{Ag}_{3}\right)$ aligned on top of cyclic peptide nanotubes over long distances $(\mu \mathrm{m})$. Experimental and computational evidence are presented to confirm the sensitivity of SNMCs towards non-covalent interactions and their 
application for the precise positioning of molecular metal clusters at the nanoscale level.

\section{Results}

Design. Self-assembled cyclic peptide nanotubes (SCPNs) are one-dimensional supramolecular polymers formed from cyclic peptide monomers that stack on top of one another through hydrogen-bonding networks. ${ }^{27-29}$ Cyclic peptide scaffolds with an even number of amino acids and alternated chirality induce the correct planar peptide ring conformation required for hydrogen bond formation and nanotube growth. The SCPN ensemble benefits from complete control over tubular diameter and external functionalization by adjusting the number and nature of the amino acid sequence. ${ }^{28-31}$ In the amphiphilic CP-1 scaffold (Fig. 1a) $\alpha$-aminoacids alternate with non-natural 3 -aminocyclopentanecarboxylic acids $\left(\gamma\right.$-Acp).$^{29,30}$ Ion pairing between orthogonal cationic (lysine) and anionic (glutamate) residues should enhance the stability of the putative hydrogen bonded antiparallel $\beta$-sheets between contiguous peptide rings. ${ }^{32}$ On the other hand, the arginine residue enhances the solubility in water and offers a perfect anchoring motif for anionic surfaces such as mica. ${ }^{29,33}$ In addition, the mica/arginine pairing assists the propagation along the surface of the $\mathrm{CP}$ self-assembly. The pyrene-modified lysine, placed at the opposite side of the arginine, helps nanotube preorganization through hydrophobic interactions in an aqueous environment. The proposed self-assembly generates ditopic ensembles that predispose cationic hydrophilic side chains towards the mica and these are confronted with the aromatic flexible arms (Fig. 1b). ${ }^{29}$

Silver clusters dispersed in water were prepared by a modified electrochemical protocol and they were free of any ligand and $\mathrm{Ag}^{+}$ions. ${ }^{2-4,7,9,10,22,34}$ The absence of $\mathrm{Ag}^{+}$ions, achieved by precipitation $(\mathrm{AgCl})$, produces highly stable clusters dispersions under standard conditions (aqueous solutions, room temperature). ${ }^{4,34}$ The UV absorbance and fluorescence emission spectra of these cluster dispersions is shown in Fig. S1. The emission shows the presence of a main band located at $3.5 \mathrm{eV}(\sim$

$350 \mathrm{~nm}$ ). According to the Jellium model, which seems to be a very good approximation for small cluters. ${ }^{35}$ The number of atoms, $\mathrm{N}$, of the cluster can be calculated by the simple expression $\mathrm{N}=\left(\mathrm{E}_{\mathrm{Fermi}} / \mathrm{E}_{\mathrm{Gap}}\right)^{3}$, where $\mathrm{E}_{\mathrm{Gap}}$ is the band gap energy of the cluster (assumed to be similar to the fluorescence emission energy) and the $\mathrm{E}_{\mathrm{Fermi}}$ is the Fermi energy for bulk silver (5.4 eV). ${ }^{33}$ From this relation one can deduce the main abundance of $\mathrm{Ag}_{3}$ clusters. The presence of another less intense peak at $4.1 \mathrm{eV}$ $(\sim 300 \mathrm{~nm})$ (Fig. S1) and a small shoulder above $3.1 \mathrm{eV}(\sim 400$ $\mathrm{nm})$, indicates also the existence of lower amounts of smaller $\mathrm{Ag}_{2}$ and larger (i.e. $\mathrm{Ag}_{4}$ ) clusters in the dispersions (Fig. S1). It can be then concluded that the clusters dispersions employed in this study are highly enriched in $\mathrm{Ag}_{3}{ }^{36}$ In addition, the high stability of these low atomicity Ag clusters under the typical experimental conditions used in this work (aqueous dispersion at room temperature) have been recently clearly established. ${ }^{34}$

DFT calculations have confirmed three equivalent $\mathrm{C}_{2 \mathrm{v}}$ obtuse isosceles triangle minimum geometries for the molecular shape of $\mathrm{Ag}_{3}$ clusters..$^{29,37,38}$ This configuration yields triangular clusters of three silver atoms without net charge. Deposition of aqueous solutions of silver clusters (enriched in $\mathrm{Ag}_{3}$ ) on mica gave rise to topographies with tiny spots that were 300-400 pm in height ( 1 atom thickness, Fig. S2) along with larger cluster aggregates that could reach a height of 2 or even $3 \mathrm{~nm}$ (Fig. S2).

SNMCs/SCPNs self-assembled hybrid architectures. CP-1 has a strong tendency to self-assemble into nanotubes, as evidence by IR, fluorescence emission and AFM topography images. ${ }^{3,29}$ Drop casting of a CP-1 aqueous solution $(100 \mu \mathrm{M}$, $\mathrm{pH} \sim 2.5$ ) on mica surfaces followed by immediate washing, under a strong flow of Milli-Q water, afforded tubular nanometric ensembles with an average height of $\sim 2.3 \mathrm{~nm}$ and lengths in the micron scale (Fig. 1e, Fig. 2a, Fig. S3). Interestingly, incubation of these mica substrates with $\mathrm{Ag}_{3}$ aqueous solutions showed a clear preference for the deposition of the SNMCs on top of the peptide nanotubular templates (Fig. 1f, S4). The self-assembly of silver clusters and SCPNs was

c)

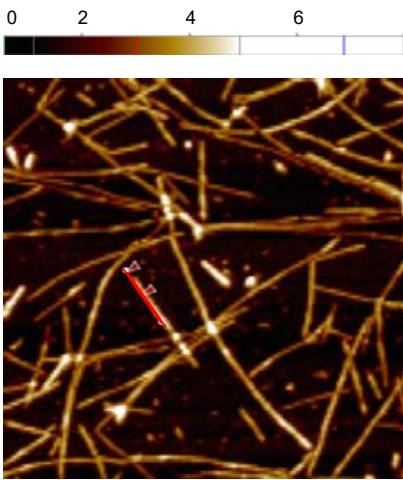

b)

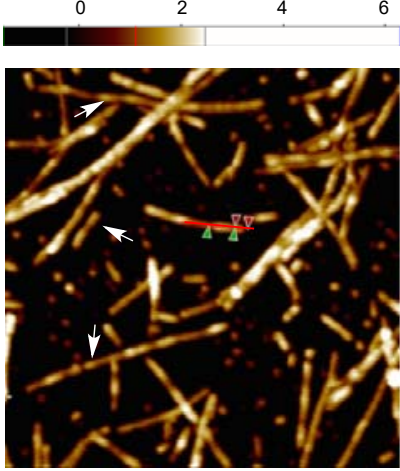

$\begin{array}{ll}0 & 2.5\end{array}$

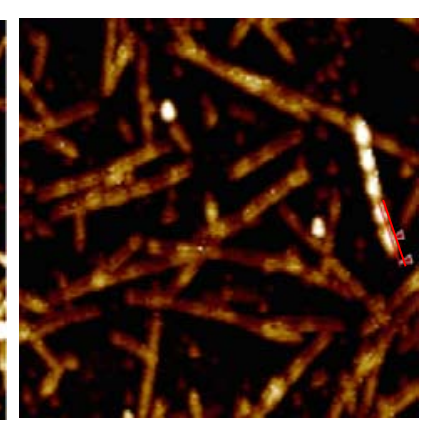

d)

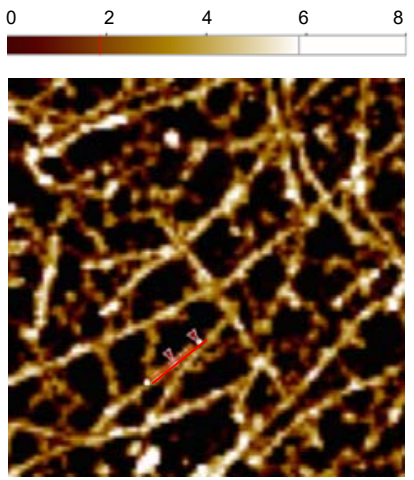

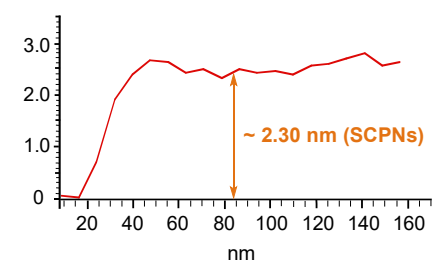
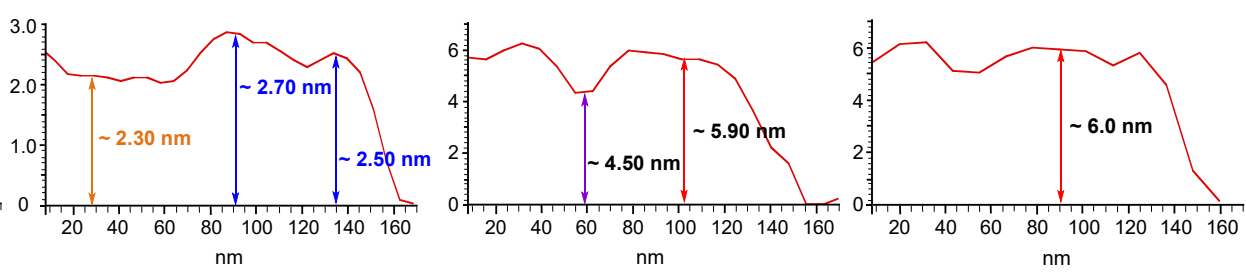

Figure 2. AFM micrographs, including topographic profiles (red lines) for increasing concentrations (and/or repeated depositions) of Ag clusters over SCPNs. a) Barely SCPNs deposited over mica (Grade V-I muscovite) from CP-1 aqueous solutions (100 $\mu \mathrm{M}$, pH $\sim 2.5$ ). The profile along the red line shows a continuous average height of $\sim 2.3 \mathrm{~nm}$. b) Silver cluster solutions enriched in $\mathrm{Ag}_{3}(10 \mu \mathrm{g} / \mathrm{mL}$ in water) self-assembled on SCPNs. The white arrows indicate height jumps below $450 \mathrm{pm}$. The corresponding profile line depicts two height jumps of 200 and 500 pm from the typical $2.3 \mathrm{~nm}$ height of individual SCPNs. c) Detail on cluster aggregates (2-3 nm) formed along peptide nanotubes. Aggregates were most frequently observed at high cluster concentration $\left(\left[\mathrm{Ag}_{3}\right]=100\right.$ or $1000 \mu \mathrm{g} / \mathrm{mL}$ in water). d) SCPNs shielded with $\mathrm{Ag}_{3}$ clusters with an average height of $6.0 \mathrm{~nm}$. Complete coverage was observed when depositions of high concentrated cluster solutions (100 or $1000 \mu \mathrm{g} / \mathrm{mL})$ were repeated for 2 or 3 times. All micrographs are $1 \mathrm{x} 1 \mu \mathrm{m}^{2}$. All scale bars are in $\mathrm{nm}$. 

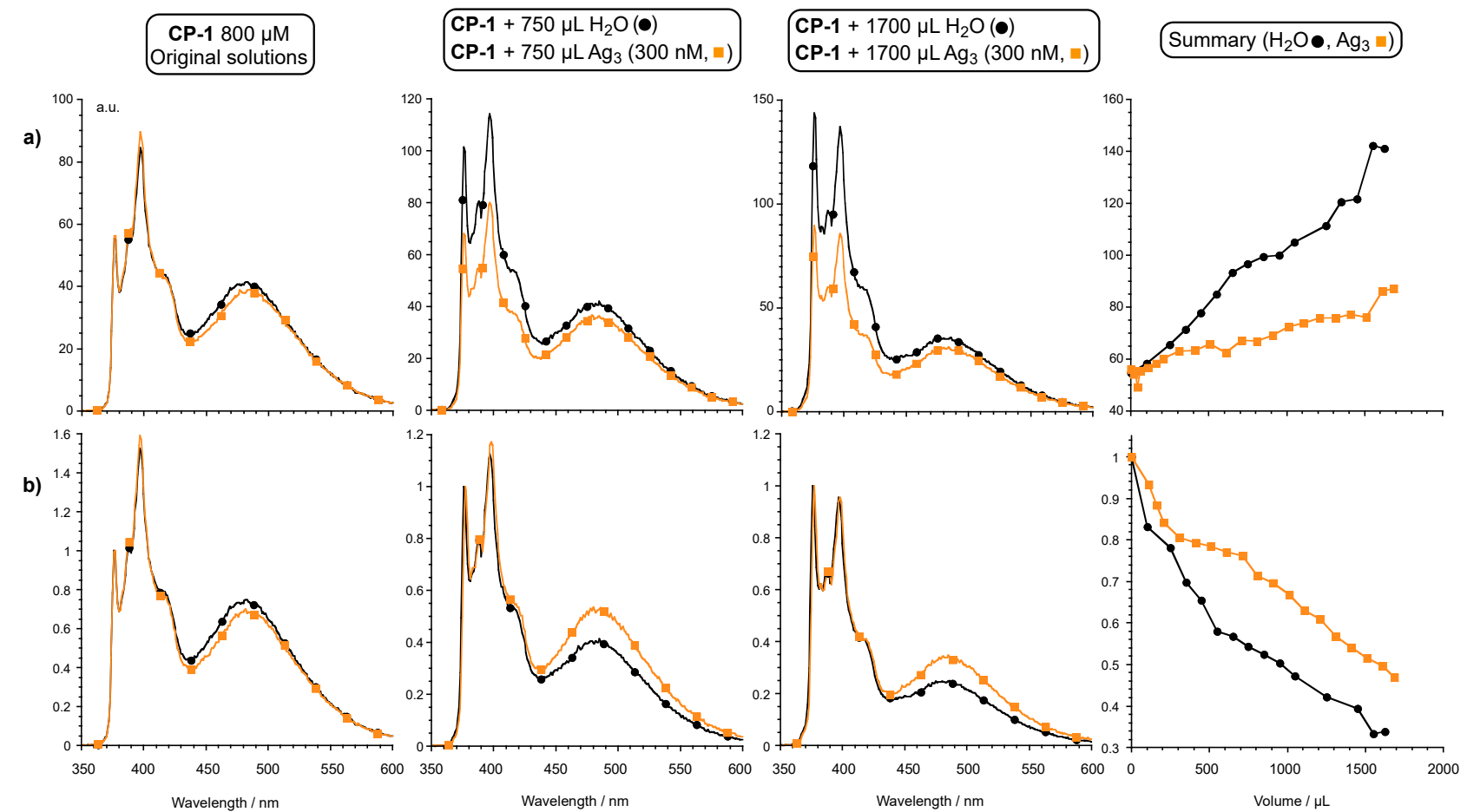

Figure 3. Pyrene fluorescence crude emission (a) and normalized emission (b) of $\mathrm{CP} 1$ solutions titrated with increasing volumes of $\mathrm{H}_{2} \mathrm{O}$ and silver clusters. The fluorescence emission was obtained by pyrene excitation $\left(\lambda_{\text {excitation }}=340 \mathrm{~nm}\right)$ and recording the emission spectra between 350 and $600 \mathrm{~nm}$. a) Pyrene crude fluorescence emission of $\mathbf{C P}-\mathbf{1}$ aqueous solution $(\mathbf{8 0 0} \boldsymbol{\mu M})$ after titration with equal volumes of $\mathrm{H}_{2} \mathrm{O}(\bullet)$ and $\mathrm{Ag}_{3}$ clusters $(300$ $\mathrm{nM})(\mathbf{\square})$ shown in black and orange respectively: a) $0 \mu \mathrm{L}, 750 \mu \mathrm{L}, 1700 \mu \mathrm{L}$ and summary at $\lambda_{\text {emission }}=373 \mathrm{~nm}$ of complete titration (Fig. S9). b) Normalized (at $373 \mathrm{~nm}$ ) pyrene fluorescence emission of $\mathbf{C P}-\mathbf{1}$ aqueous solution after titration with $\mathrm{H}_{2} \mathrm{O}(\bullet)$ and $\mathrm{Ag}_{3}$ clusters (300 nM) (Ш): $0 \mu \mathrm{L}, 750$ $\mu \mathrm{L}, 1700 \mu \mathrm{L}$ and normalized summary at $\lambda_{\text {emission }}=470 \mathrm{~nm}$ of complete titration. In b) the normalization of the crude fluorescent emission data was at $\mathrm{I}_{1}$ $=373 \mathrm{~nm}$ and in the titration summary the initial maximum was normalized to 1 (Fig. S10).

observed either in sequential depositions or when $\mathbf{C P}-\mathbf{1}$ and $\mathrm{Ag}_{3}$ clusters were previously premixed in water and deposited together (see Methods). As depicted in Fig. 1b, the natural organization of CP-1 into nanotubes, directed by the arginine side chain/mica interactions, should lead to the alignment of aromatic pyrene residues away from the surface. In this arrangement, the preferential cluster alignment over the nanotubular longitudinal axis should be a consequence of cluster- $\pi$ interactions between the planar pyrene moiety and the triangular $\mathrm{Ag}_{3}$ clusters. It should be noted that the small size of SNMCs, such as $\mathrm{Ag}_{3}$, implies that they are much less prone to form covalent bonds with other functional groups. ${ }^{3,29}$ Despite the short height of the planar single clusters (300-450 pm depending on orientation) and the resolution of the AFM Z scanner (25 pm per pixel) it was possible to identify differences in the nanotube surface profiles that can be assigned to single clusters $(<450 \mathrm{pm})$ in the AFM topographic images (Fig. 2b, Fig S5). In these figures ( $2 \mathrm{~b}$ and $\mathrm{S} 5$ ), several discontinuous height jumps within the range of 200-450 pm (white arrows) were assigned to single $\mathrm{Ag}_{3}$ clusters settling on top of peptide nanotubes. Comparison of the AFM micrographs in figures $2 \mathrm{a} / 2 \mathrm{~b}$ and figure $\mathrm{S} 2$ shows no cluster preferential deposition and/or aggregation in the naked mica surface partially covered by SCPNs. The amount of silver clusters deposited along peptide nanotubes increases with increasing the concentration of the cluster solutions employed (Fig. 2c). Interestingly, successive depositions of concentrated $\mathrm{Ag}_{3}$ afforded complete silver coverage $(\sim 6 \mathrm{~nm})$ of the peptide tubular cores (Fig. 2d, Fig. S6). Control experiments with cyclic peptides lacking the pyrene aromatic moieties confirmed the absence of preferential deposition of silver clusters on top of these self-assembled nanotubes (Figs. S25 and S26).
Pyrene fluorescence emission and Circular Dichroism studies in $\mathbf{A g}_{3} / \mathbf{S C P N s}$ aqueous solutions. The presence of a pyrene residue provides an excellent opportunity to track the supramolecular interactions of the CP-1 rings in solution. ${ }^{29}$ The emergence of an excimer band on increasing the concentration of CP-1 unambiguously confirms pyrene stacking in solution and allows the calculation of a rough association constant of $10^{3} \mathrm{M}^{-1}$ for aqueous nanotube formation..$^{29,39}$ Tubular ensembles in solution are further supported by the instantaneous nanotubular self-assembly that takes place upon mica deposition (vide supra). In order to gain an insight into the interaction of SCPNs and $\mathrm{Ag}_{3}$ clusters in solution, stock aqueous solutions of CP-1 (of different concentrations: 1600,800 and $400 \mu \mathrm{M}$ ) were titrated, with increasing volumes of $\mathrm{Ag}_{3}$ clusters (Fig. 3, Fig. S8-S11). It should be noted that, according to the pyrene and $\mathrm{Ag}_{3}$ luminescent features, and under the concentration regime employed, the possible $\mathrm{Ag}_{3}$ background interference is negligible. The total lack of interference from the silver clusters luminescence was confirmed by measuring the same titration experiments at the lower clusters excitation wavelength ( $\lambda$ excitation $=227 \mathrm{~nm}$ ) and acquiring the emission spectra including the cluster emission gap that, as expected, showed no emission bands (Fig. S8). In the studied system, the typical concentration quenching and the possible dispersive scattering effects led to an increase in the crude fluorescence emission upon dilution of concentrated CP-1 solutions (Fig. S9). Interestingly, this increase in the emission intensity was significantly reduced when the CP-1 solutions were titrated with aliquots of $\mathrm{Ag}_{3}$ solution rather than with the same volumes of pure water (Fig. $3 a)$. We surmise that the less marked increase in emission intensity upon dilution (in the presence of SNMCs) is due to the stabilization of the SCPNs, which logically would diminish the 
amount of fully solvated CP-1 rings in solution. On the other hand, in the normalized emission spectra $(377 \mathrm{~nm})$ it was observed that the disappearance of the excimer band $\left(\lambda_{\text {emission }}=\right.$ $470 \mathrm{~nm}$ ) was noticeably delayed in the presence of silver clusters (Fig. 3b, Fig. S10). This prevalence of the excimer band should correspond to a better adjustment of pyrene aromatic rings in the presence of $\mathrm{Ag}_{3}$. Importantly, the behaviour was retained for different CP-1 starting concentrations (400, 800 and $1600 \mu \mathrm{M})$ (Fig. 3 and Fig. S11). Taken together, the drop in the crude emission and the delayed excimer quenching of CP-1 in the presence of increasing amounts of clusters of $\mathrm{Ag}_{3}$, provides a reliable evidence for the stabilization of the corresponding SCPNs by metal clusters in aqueous solution (Fig. S7).

The chiral environment of the self-assembled peptide nanotube influenced the circular polarized light absorption of the pyrene chromophore as well as the potential secondary structure of the cyclic peptide in solution. We have carried out circular dichroism (CD) titration experiments analogous to the fluorescence experiments where we treated CP1 stock solutions with either silver clusters aqueous dispersions or pure Milli-Q water. The different intensity variations in different exciton couplings of the titrated peptide, with Milli-Q water or cluster dispersions, supported the interactions between silver clusters and peptide nanotubes in solution (Fig. S12).

Computational chemistry. Using the B97D3 functional, which incorporates van der Waals corrections ${ }^{37-39}$ with the moderately large basis set $6-31+\mathrm{G}(\mathrm{d}, \mathrm{p})$ and LanL2dz for Ag, a strong $\mathrm{Ag}_{3} /$ Pyrene interaction was clearly identified (Fig. 4a). The shape of the $\mathrm{Ag}_{3}$ clusters interacting with the pyrene sandwich remained as an isosceles triangle (distances: $0.27,0.27$, $0.31 \mathrm{~nm}$ ) with the vertices surrounded by carbons of the pyrene units at short distances ranging from 0.29 to $0.62 \mathrm{~nm}$ (Fig. S14). The interaction of the $\mathrm{Ag}_{3}$ cluster with the pyrene sandwich supposes a strong stabilization of $-116.6 \mathrm{~kJ} / \mathrm{mol}$, taking into account the correction for basis set superposition error (BSSE), using the counterpoise approach (see Methods). The encapsulation of $\mathrm{Ag}_{3}$ cluster between the two pyrene units is associated with negative Gibbs energy values $(-31.4 \mathrm{~kJ} / \mathrm{mol}$, or $-2.9 \mathrm{~kJ} / \mathrm{mol}$, taking into account the BSSE). Similar results were obtained when the same initial structures were minimized in the presence of implicit water resulting into a stronger stabilization of the complex (Fig. S14).

Although DFT methods work well for single cluster and small molecules, ${ }^{24,37,38}$ molecular dynamics (MD) are suitable for the simulations of more complex and dynamic hybrid systems. ${ }^{24,40,41}$ The Lennard-Jones (LJ) parameterization for neutral metal atoms have proven to be a reliable alternative to reproduce experimental data or accurate quantum chemistry calculations at the appropriate ranges for Pauli repulsion and van der Waals attractive forces..$^{29,40,41} \mathrm{MD}$ simulations, modelling $\mathrm{Ag}(0)$ as $\mathrm{LJ}$ particles (see Methods), were performed with unrestricted pre-formed triangular $\mathrm{Ag}_{3}$ clusters, at a high concentration (200 $\mathrm{mM}$ ), in the presence of a discrete solvated SCPN made of ten cyclic peptide monomers (Fig. 4b). After 10 ns of MD simulations the self-assembly of $\mathrm{Ag}_{3}$ clusters was confirmed over the hydrophobic pyrene side of the nanotube (Fig. 4b). The calculations showed that the metal clusters mainly interact with the pyrene moieties. In addition, we could also observe interactions of the silver clusters with the heteroatoms of the amide functional group $(\mathrm{N}, \mathrm{O})$ in close proximity to the pyrene aromatic ring. These binding sites worked as nucleation centres for the growth of larger cluster aggregates, which is consistent with the experimental AFM results (Fig. 2c-d). Internalization of silver clusters in the nanotube lumen was not observed in any case. Forced encapsulation of $\mathrm{Ag}_{3}$ inside the SCPN led to a significant distortion of the channel (Fig. S15). The interaction between $\mathrm{Ag}_{3}$ and SCPNs is translated into an increase of the
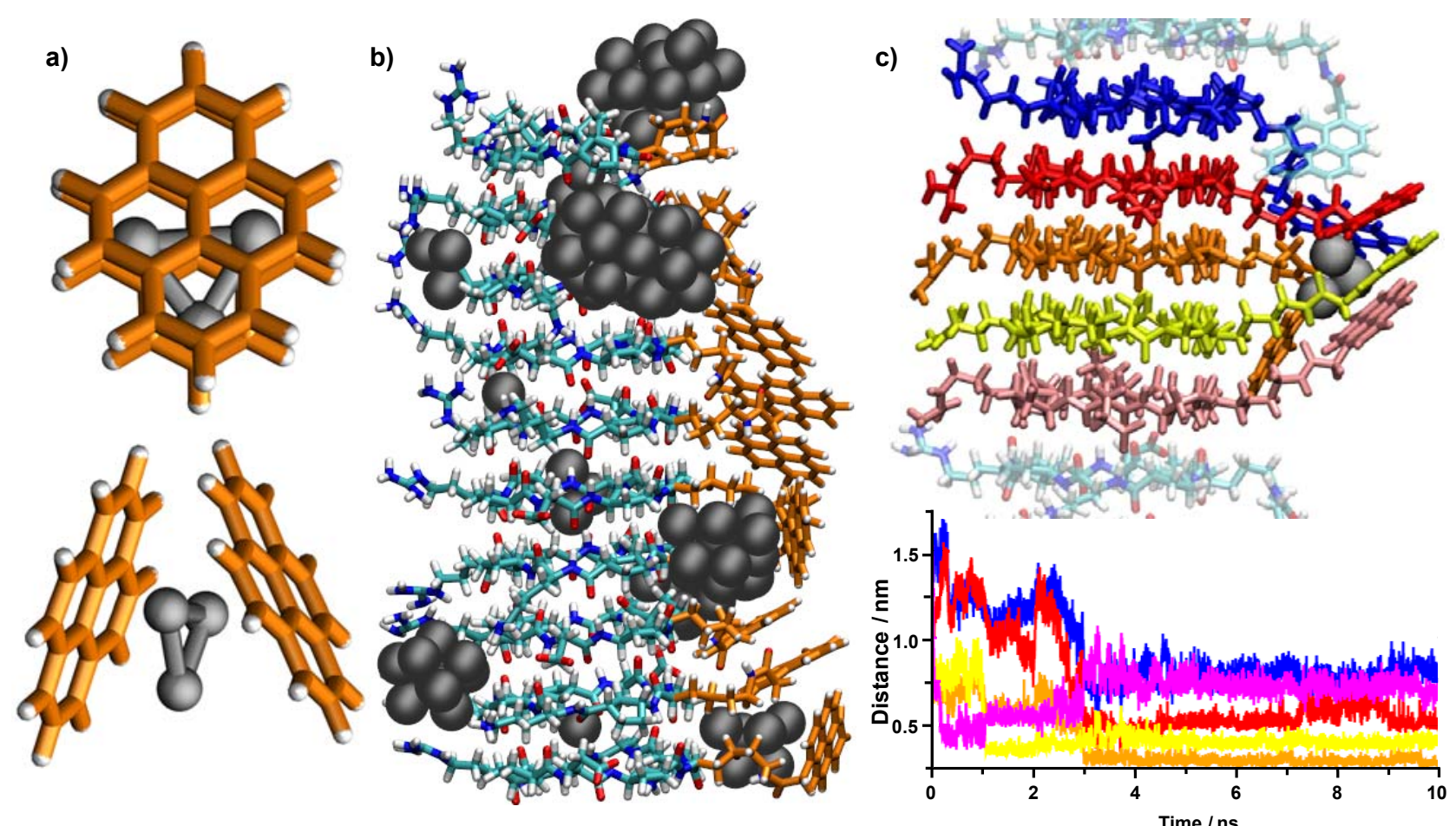

Figure 4. Computational chemistry. a) DFT calculations [B97D3/6-31+G(d,p) and LanL2dz for Ag] for one $\mathrm{Ag}_{3}$ cluster and two pyrene molecules. b) Snapshot structure at $\mathrm{t}=10 \mathrm{~ns}$ after MD simulation of the SCPN in the presence of single $\mathrm{Ag}_{3}(200 \mathrm{mM})$ showing preferential cluster accumulation along the hydrophobic pyrene side of the nanotube. c) Detail of a snapshot of the surroundings of one $\mathrm{Ag}_{3}$ cluster self-assembled in the middle of a peptide nanotube $(10 \mathrm{CPs})$ after $10 \mathrm{~ns}$ of MD simulations. The graph depicts the distances between the centres of mass of $\mathrm{Ag}_{3}$ and pyrene moieties in the computed ensemble during the simulation time. Each CP ring of the SCPN is represented by the same colour in the tubular model and in the 2D graph. 
interaction energy between the different $\mathrm{CP}$ units in several regions of the nanotube (Fig. S16). The energy difference $(\Delta \mathrm{E})$, for the interactions between contiguous CPs of a simulated nanotube is favourable in the presence of $\mathrm{Ag}_{3}$ (Fig S16, CPs 3-4 and 5-6).

The hybrid ensemble was further investigated by performing MD simulations starting from a solvated SCPN in the presence of two $\mathrm{Ag}_{3}$ clusters (Fig. S17; Video S1). Both the nanotube and the clusters, which were simulated without any restriction, were stable all along the simulation time (10 ns). Interestingly, at a fixed radial distance of $0.5 \mathrm{~nm}$ the large number of contacts (123-152) between the cluster and peptides resulted in a minimum distance of around $0.2-0.3 \mathrm{~nm}$ between the $\mathrm{Ag}_{3}$ and the nanotube (Fig. S17). During these simulations it was noticed that almost all of the cyclic monomers are involved, at least to some extent, in the interaction with the metal centres (Fig. S18). Therefore, the interaction energy between the cluster and the SCPN, which is dominated by the short-range LJ term, decreased very rapidly during the first steps of the trajectory and reached quite stable minimums in the range of $-250 \mathrm{~kJ} / \mathrm{mol}$ (Fig. S18). Taking into account the number of $\mathrm{CPs}$ in the calculated ensemble this energy represents a value of $\sim 40 \mathrm{~kJ} / \mathrm{mol}$ for each $\mathrm{CP}$ unit (1 pyrene-1 $\left.\mathrm{Ag}_{3}\right)$, which is in good agreement with the DFT counterpoise complexation energies obtained with the pyrene model $(\sim-50 \mathrm{~kJ} / \mathrm{mol}$ for each pyrene/cluster sandwich Fig. 4a, S14). Furthermore, MD simulations showed that the pyrenes were able to encapsulate $\mathrm{Ag}_{3}$ clusters, thus affording 'pyrene jails' for metal clusters (Fig. 4c). Four to five pyrenes are positioned at a range of $1 \mathrm{~nm}$ around each of the silver clusters (Fig. 4c, S18 and S19). In this arrangement it was also possible to identify silver contacts with the heteroatoms (N/O) of the amide linker (Figs. S19 and S20). It was remarkable to observe complete dehydration of one of the silver clusters, indicating that pyrene is able to completely replace the first solvation sphere of $\mathrm{Ag}_{3}$ clusters in aqueous solution (Fig. S21). The importance of the solvent was highlighted when MD simulations were carried out in chloroform and toluene. Different starting structures of $\mathrm{Ag}_{3} / \mathrm{SCPN}$ disclosed the expulsion of the metal clusters from the pyrene coordination sphere of the hybrid $\mathrm{Ag}_{3} / \mathrm{SCPN}$ ensemble in hydrophobic solvents (Figs. S22 and S23).

To address the potential interactions of other minor components of the clusters dispersions we have carried out the same DFT calculations for clusters composed of 2 and 4 silver atoms. Quantum mechanic calculations in the gas phase showed favourable enthalpic interactions between silver clusters molecules of small atomicity $\left(\mathrm{Ag}_{2}, \mathrm{Ag}_{4}\right)$ and pyrene sandwiches (Fig. S24, Table S1). These enthalpic contributions increased with increasing number of silver atoms in the cluster (Fig. S24, Table S1). However, the positive value of the free Gibbs energy, obtained for $\mathrm{Ag}_{2}$ clusters, suggests an unfavourable interaction of the pyrene moieties with the second most abundant component of our cluster dispersions (Table S1).

\section{Discussion}

We here report the sensitivity of neat metal clusters to non-covalent interactions and the application of these interactions to the preparation of self-assembled 1D architectures of $\mathrm{Ag}_{3}$ clusters on top of peptide nanotubes.

According to our design principles, the peptide scaffold is anchored onto the anionic mica through a cationic arginine residue that affords nanotubes with hydrophobic aromatic pyrene residues aligned orthogonally to the hydrophilic surface (Figs. 1a-c). ${ }^{29,42}$ Concomitant or sequential deposition (over mica) of $\mathbf{C P - 1} / \mathrm{Ag}_{3}$ mixtures afforded clear 1-D self-assembled metal clusters along the nanotube longitudinal axis (Fig. 1e,f, Fig.
S4). In the observed long distance ( $\mu \mathrm{m})$ hierarchical architecture it was possible to identify single clusters, as evidenced by short height increments $(<0.45 \mathrm{~nm})$ frequently observed in topographic micrographs on top of the SCPN (Fig. 2b and profile). Interestingly, cluster aggregation (also observed for pure $\mathrm{Ag}_{3}$ clusters, Fig. S2) retained the preference towards the nanotubular scaffolds (Fig. 2c). The use of increased concentrations and/or repeated deposition of SNMCs provided attractive hybrid 'nanowire like' structures composed of metal silver cluster coatings of inner organic tubular peptide cores (Fig. 2d).

Solution fluorescence experiments, in where SCPNs were titrated with $\mathrm{Ag}_{3}$ clusters, confirmed the existence of non-covalent interactions and the synergic stabilization of the peptide/metal supramolecular aggregates. This stabilization of the self-assembled tubular architectures might be rationalized by the cluster intercalation between the pyrene excimers (Fig. S20). The inter-pyrene distance was estimated to be around $3.5 \AA^{31,42}$ while the peptide-peptide distance in the antiparallel $\beta$-sheet characteristic of peptide nanotubes is around $4.7 \AA .1,5,8,12,18-21,31$ This disparity in the distance constrains the pyrene stacking and forces some hydrophobic surfaces to be exposed to water, a situation that might hinder nanotube growth. The incorporation of $\mathrm{Ag}_{3}$ clusters between pyrenes every few residues might adjust the aromatic ring distances to the backbone-backbone separation and this may help to facilitate and stabilize the self-assembly of the hybrid nanotube (Fig. S7).

DFT and MD methods supported the proposed mode of interaction between $\mathrm{Ag}_{3}$ clusters and hydrophobic pyrene motifs. Molecular dynamic simulations of unrestricted peptide/metal mixtures showed a preferential deposition and/or aggregation of $\mathrm{Ag}_{3}$ clusters within the pyrene hydrophobic coordination sphere. This picture (Fig. 4B) clearly shows the agreement of the computational calculations with the experimental AFM images, where arginine moieties anchor the peptide nanotube in the mica surface and silver clusters are deposited within the pyrene aromatic arms. The slightly higher interaction energy calculated for cluster-decorated nanotubes suggested a stabilization of the SCPNs in the presence of triatomic silver clusters. The calculated solvent dependence on the stability of the $\mathrm{Ag}_{3} / \mathrm{SCPN}$ hybrids also supports the importance of the hydrophobic effect and van der Waals interactions.

Different templates have been applied in the size-controlled synthesis of metal clusters. . $^{1,5,8,12,18-21}$ In biopolymer templated protocols, the cluster size selection was achieved by the coordination of the anionic DNA structures to metal salts during chemical reduction of the cationic silver $\left(\mathrm{Ag}^{+}\right) .^{1,5,20,23}$ In other words, the uncontrolled reductive nucleation of the metal particles is modulated according to the corresponding template (e.g. DNA). However, in the strategy reported here, the size and the properties of the metal cluster are previously fixed by controlled synthesis and purification, and it is the supramolecular host who adjust its structure to maximize non-covalent interactions and stabilize the hybrid architecture. Furthermore, this strategy enables the precise alignment of molecular metal clusters free of superfluous surfactants, salts, buffers or chemical reagents and outlines the potential usefulness of other non-ionic supramolecular templates.

The observed solvatochromism ${ }^{23,24}$ and the calculated cluster- $\pi$ stacking in DNA composites ${ }^{24,34}$ highlighted the important roles of hydrophobic effects and dipole (or quadrupole) induction in the modulation of the nanocluster environment and the stability of their supramolecular complexes. Furthermore, the intercalation of silver clusters within DNA 
double helices has been postulated and studied by UV spectroscopy. ${ }^{17,34}$ However, non-covalent interactions of molecular metal clusters of zero valence have not been seen at work. The results reported here highlight the importance that supramolecular chemistry and non-covalent interactions will play in the emerging field of subnanometric metal (0) clusters. In this work we have applied this undisclosed concept in the preparation of self-assembled hybrid architectures where $\mathrm{Ag}_{3}$ clusters are aligned over long distances $(\mu \mathrm{m})$ on top of peptide nanotubes. The design principles and the observed experimental and computational results imply that the interactions between peptide nanotubes and the subnanometric metal $(0)$ clusters arise from the establishment of cluster- $\pi$ dipole induced interactions between silver clusters and the planar aromatic pyrene motifs. The findings reported here pave the way for the extensive investigation, identification and modulation of novel non-covalent interactions between pure SNMCs and supramolecular hosts. In this respect, the direct coordination of multiaromatic surfaces (i.e. pyrenes or other cores: antracene, naphthalenediimides, perylenes, etc) offer a very attractive alternative complementary to the classical carbon monoxide ligands. ${ }^{17}$ It is essential to note that this type of interactions would not only depend on the structure of the supramolecular host and the solvent environment, but also on the features of the SNMC, which in turn are controlled by several factors such as the nature of the metal, number of atoms, size, shape, band gap, polarizability, etc. It is important to note that the same kind of interactions could be operative and therefore applicable to other silver clusters of small atomicity. ${ }^{14,15,34}$ Complementary to the central importance of synthetic protocols, the supramolecular and non-covalent chemistry of SNMCs will enable innovative approaches to be developed for the characterization, manipulation and application of these promising molecular metal clusters.

\section{Conclusion}

To summarize we report the sensitivity of molecular-like subnanometric metal (0) clusters towards non-covalent interactions. In this work we have applied these interactions for the synergic self-assembly of $\mathrm{Ag}_{3}$ and silver clusters of small atomicity with peptides nanotubes. Topographic micrographs of concomitant or sequential deposition of SCPNs and $\mathrm{Ag}_{3}$ over mica revealed a clear preference of silver SNMCs to be deposited and aligned on top of the peptide nanotubes. AFM topographic images of increasing concentrations of silver clusters showed that the preference was maintained from low dosages to high concentrated and/or repeated silver depositions. Fluorescence studies of solutions of SCPNs titrated with the $\mathrm{Ag}_{3}$ disclosed the presence of the self-assembled supramolecular structures where each member of the ensemble stabilizes the other in aqueous solution. Computational calculations with DFT and molecular dynamics supported the proposed pyrene/cluster type of interactions. The findings reported here constitute the first example of the establishment and the application of non-covalent interactions between neat metal-atom clusters (with zero valence) and a supramolecular counterpart. In the long term, these results outline the possibilities of this type of approach to exploit the great properties of neat discrete metal clusters combined with supramolecular hosts. Our efforts in this direction will be reported in due course.

\section{Methods}

AFM of self-assembled $\mathrm{Ag}_{3}$ clusters on SCPNs.
The $\mathrm{pH}$ of purified stock solutions of CP-1 $(200 \mu \mathrm{M}$ or $100 \mu \mathrm{M})$ in Milli-Q water was adjusted to $\sim 2.5$ with TFA $(100 \mathrm{mM}$ in Milli-Q water). Silver clusters dispersed in water $(1 \mathrm{mg} / \mathrm{L})$ were purchased from Nanogap, Spain (NGAPAQC Ag-1102-W) and were used without further purification. The starting silver cluster (enriched in $\mathrm{Ag}_{3}$ ) stock solution was diluted with Milli-Q water to $100 \mu \mathrm{g} / \mathrm{L}$ or $10 \mu \mathrm{gr} / \mathrm{L}$. The general protocol for deposition onto a mica substrate: Stock solutions were drop-casted over freshly exfoliated mica sheets (SPI Supplies, grade V-1 Muscovite), immediately washed with copious amounts of Milli-Q water, and dried under a flow of nitrogen. Protocol (a) CP-1 stock solutions were deposited, washed and dried. The resulting nanotube covered micas were again drop casted with $\mathrm{Ag}_{3}$ clusters, washed and dried. Protocol b) CP-1 and $\mathrm{Ag}_{3}$ clusters were sequentially drop-casted over mica, washed and dried. Protocol (c) aliquots of CP-1 and $\mathrm{Ag}_{3}$ stock solutions were mixed and incubated for $5 \mathrm{~min}$. The mixture was deposited washed and dried. Standard AFM measurements were conducted under ambient atmosphere at room temperature using a XE-100 instrument (Park Systems Corporation) in non-contact mode. The high-resonance non-contact AFM cantilever (ACTA probe, $\square=330 \mathrm{kHz}$ ). Average topographic height values for selected micrographs are given in Fig. S13.

\section{Fluorescence spectroscopy titrations.}

CP-1 stock solutions at different concentrations (1600, 800, 400 $\mu \mathrm{M})$ were titrated with increasing volumes $(0-2 \mathrm{~mL})$ of either Milli-Q water or $\mathrm{Ag}_{3}$ aqueous stock solutions $(300 \mathrm{nM})$. The $\mathrm{pH}$ of each sample was below 3. Steady-state fluorescence measurements were carried out in a Jobin-Yvon Fluoromax-3 (DataMax 2.20) system, coupled to a temperature controller (Wavelength Electronics LFI-3751), and using a standard Hellman semi-micro cuvette (114F-QS, $10 \mathrm{~mm}$ light path). Titrations were carried out at $25^{\circ} \mathrm{C}$ and $\lambda$ excitation $=340 \mathrm{~nm}$.

Computational chemistry (see supporting information). MD simulations were performed with the GROMACS 4.6 Molecular Dynamics package. The GAFF force-field was employed for the SCPN and the neutral atoms were modelled as Lennard-Jones (LJ) particles (supporting information). The LJ parameters for the metal clusters have been used previously to study the growth of $\mathrm{Ag}$ film ${ }^{40}$ and the interaction between $\mathrm{Ag}_{12}$ clusters with single-stranded DNA. ${ }^{24}$ For initial MD simulations $\mathrm{Ag}_{3}$ concentration was fixed at $200 \mathrm{mM}$ in order to favour the $\mathrm{CP} / \mathrm{Ag}_{3}$ and cluster/cluster intermolecular interactions in the short time scale. MD simulations were repeated using another set of parameters developed by Heinz, ${ }^{41}$ leading to very similar results. All of the DFT calculations reported in this study were performed with the Gaussian 09 package (see supporting information).

Suporting information. Full experimental and computational details can be found online in the supporting information. This material is available free of charge via the Internet at http://pubs.acs.org.

\section{References}

(1) Berti, L.; Alessandrini, A.; Facci, P. DNA-

Templated Photoinduced Silver Deposition. J. Am. Chem. Soc. 2005, 127, 11216-11217.

Häkkinen, H. Atomic and Electronic Structure of Gold Clusters: Understanding Flakes, Cages and Superatoms From Simple Concepts. Chem. Soc. Rev. 2008, 37, 1847-1859.

(3) Corma, A.; Concepción, P.; Boronat, M.; Sabater, M. J.; Navas, J.; Yacaman, M. J.; Larios, E.; Posadas, A.; 
López-Quintela, M. A.; Buceta, D. Exceptional Oxidation Activity with Size-Controlled Supported Gold Clusters of Low Atomicity. Nat. Chem. 2013, 5, 775-781.

(4) Lu, Y.; Chen, W. Sub-Nanometre Sized Metal Clusters: From Synthetic Challenges to the Unique Property Discoveries. Chem. Soc. Rev. 2012, 41, 3594-3623.

Copp, S. M.; Schultz, D. E.; Swasey, S.; Gwinn, E. G. Atomically Precise Arrays of Fluorescent Silver Clusters: a Modular Approach for Metal Cluster Photonics on DNA Nanostructures. Acs Nano 2015, 9, 2303-2310.

(6) Zhang, Y.; Dai, H. Formation of Metal Nanowires on Suspended Single-Walled Carbon Nanotubes. Appl. Phys. Lett. 2000, 77, 3015-3017.

(7) Peyser, L. A.; Vinson, A. E.; Bartko, A. P.; Dickson, R. M. Photoactivated Fluorescence From Individual Silver Nanoclusters. Science, 2001, 291, 103-106.

(10) Attia, Y. A.; Buceta, D.; Blanco-Varela, C.;

Mohamed, M. B.; Barone, G.; López-Quintela, M. A. Structure-Directing and High-Efficiency

Photocatalytic Hydrogen Production by Ag Clusters.

J. Am. Chem. Soc. 2014, 136, 1182-1185.

(11) Moscatelli, A. The Struggle for Control. Nat.

Nanotech. 2013, 8, 888-890.

(12) Correa-Duarte, M. A.; Pérez-Juste, J.;

Sánchez-Iglesias, A.; Giersig, M.; Liz-Marzán, L. M. Aligning Au Nanorods by Using Carbon Nanotubes as Templates. Angew. Chem. Int. Ed. 2005, 44, 4375-4378.

(13) Alves, L.; Ballesteros, B.; Boronat, M.;

Cabrero-Antonino, J. R.; Concepción, P.; Corma, A.; Correa-Duarte, M. A.; Mendoza, E. Synthesis and Stabilization of Subnanometric Gold Oxide Nanoparticles on Multiwalled Carbon Nanotubes and Their Catalytic Activity. J. Am. Chem. Soc. 2011, 133, 10251-10261.

(14) Reuter, T.; Vidoni, O.; Torma, V.; Schmid, G.; Nan, L.; Gleiche, M.; Chi, L.; Fuchs, H. Two-Dimensional Networks via Quasi One-Dimensional Arrangements of Gold Clusters. Nano Lett. 2002, 2, 709-711.

(15) Hoeppener, S.; Chi, L.; Fuchs, H. Formation of Au5s Strands on a Molecular Template at the Solid-Liquid Interface. Nano Lett. 2002, 2, 459-463.

(16) Liu, Y.; Male, K. B.; Bouvrette, P.; Luong, J. H. T. Control of the Size and Distribution of Gold Nanoparticles by Unmodified Cyclodextrins. Chem. Mater. 2003, 15, 4172-4180.

(17) Gates, B. C. Supported Metal Clusters: Synthesis, Structure, and Catalysis. Chem. Rev. 1995, 95, 511-522.

(18) Petty, J. T.; Zheng, J.; Hud, N. V.; Dickson, R. M. DNA-Templated Ag Nanocluster Formation. J. Am. Chem. Soc. 2004, 126, 5207-5212.

(19) Guo, W.; Yuan, J.; Dong, Q.; Wang, E. Highly Sequence-Dependent Formation of Fluorescent Silver Nanoclusters in Hybridized DNA Duplexes for Single Nucleotide Mutation Identification. J. Am. Chem. Soc. 2010, 132, 932-934.

Timper, J.; Gutsmiedl, K.; Wirges, C.; Broda, J.;
(21) O’Neill, P. R.; Young, K.; Schiffels, D.; Fygenson, D. K. Few-Atom Fluorescent Silver Clusters Assemble at Programmed Sites on DNA Nanotubes. Nano Lett. 2012, 12, 5464-5469.

(22) Selva, J.; Martínez, S. E.; Buceta, D.; RodríguezVázquez, M. J.; Blanco, M. C.; López-Quintela, M. A.; Egea, G. Silver Sub-Nanoclusters Electrocatalyze Ethanol Oxidation and Provide Protection Against Ethanol Toxicity in Cultured Mammalian Cells. $J$. Am. Chem. Soc. 2010, 132, 6947-6954.

(23) Vilar-Vidal, N.; Blanco, M. C.; López-Quintela, M. A.; Rivas, J.; Serra, C. Electrochemical Synthesis of Very Stable Photoluminescent Copper Clusters. J. Phys. Chem. C 2010, 114, 15924-15930.

(24) Samanta, P. K.; Periyasamy, G.; Manna, A. K.; Pati, S. K. Computational Studies on Structural and Optical Properties of Single-Stranded DNA Encapsulated Silver/Gold Clusters. J. Mater. Chem. 2012, 22, 6774-6781.

(25) Aradhya, S. V.; Frei, M.; Hybertsen, M. S.; Venkataraman, L. Van Der Waals Interactions at Metal/Organic Interfaces at the Single-Molecule Level. Nat. Mater. 2012, 11, 872-876.

Sony, P.; Puschnig, P.; Nabok, D.; Ambrosch-Draxl, C. Importance of Van Der Waals Interaction for Organic Molecule-Metal Junctions: Adsorption of Thiophene on $\mathrm{Cu}(110)$ as a Prototype. Phys. Rev. Lett. 2007, 99, 176401-176404.

Brea, R. J.; Reiriz, C.; Granja, J. R. Towards Functional Bionanomaterials Based on Self-Assembling Cyclic Peptide Nanotubes. Chem. Soc. Rev. 2010, 39, 1448-1456.

Montenegro, J.; Ghadiri, M. R.; Granja, J. R. Ion Channel Models Based on Self-Assembling Cyclic Peptide Nanotubes. Acc. Chem. Res. 2013, 46, 2955-2965.

Montenegro, J.; Vázquez-Vázquez, C.; Kalinin, A.; Geckeler, K. E.; Granja, J. R. Coupling of Carbon and Peptide Nanotubes. J. Am. Chem. Soc. 2014, 136, 2484-2491.

Reiriz, C.; Brea, R. J.; Arranz, R.; Carrascosa, J. L.; Garibotti, A.; Manning, B.; Valpuesta, J. M.; Eritja, R.; Castedo, L.; Granja, J. R. $\alpha, \gamma$-Peptide Nanotube Templating of One-Dimensional Parallel Fullerene Arrangements. J. Am. Chem. Soc. 2009, 131, 11335-11337.

Amorin, M.; Castedo, L.; Granja, J. R. New Cyclic Peptide Assemblies with Hydrophobic Cavities: the Structural and Thermodynamic Basis of a New Class of Peptide Nanotubes. J. Am. Chem. Soc. 2003, 125, 2844-2845.

Pérez-Alvite, M. J.; Mosquera, M.; Castedo, L.; Granja, J. R. Toward the Rational Design of Molecular Rotors Ion Sensors Based on $\alpha, \gamma$-Cyclic Peptide Dimers. Amino Acids 2011, 41, 621-628. Zheng, J.; Zhang, C.; Dickson, R. Highly Fluorescent, Water-Soluble, Size-Tunable Gold Quantum Dots. Phys. Rev. Lett. 2004, 93, 077402-077406.

Buceta, D.; Busto, N.; Barone, G.; Leal, J. M.; Domínguez, F.; Giovanetti, L. J.; Requejo, F. G.; García, B.; López-Quintela, M. A. $\mathrm{Ag}_{2}$ and $\mathrm{Ag}_{3}$ Clusters: Synthesis, Characterization, and Interaction with DNA. Angew. Chem. Int. Ed. 2015, 54, 7612-7616. 
(35) Santiago-González, B.; López-Quintela, M. A. New Strategies and Synthetic Routes to Synthesize Fluorescent Atomic Quantum Clusters. In Functional Nanometer Sized Clusters of transition Metals: Synthesis and Applications; Chen, W.; Chen, S., Eds.; The Royal Society of Chemistry: Cambridge, 2014; pp. 25-50.

(36) However, the conclusions of this study should not be restricted to a particular size of clusters and could be extrapolated to other silver clusters of small atomicity (i.e. $\mathrm{Ag}_{2}$ and $\mathrm{Ag}_{4}$ ).

(37) Chen, M.; Dyer, J. E.; Li, K.; Dixon, D. A. Prediction of Structures and Atomization Energies of Small Silver Clusters, $(\mathrm{Ag})_{n}, n<100$. J. Phys. Chem. A 2013, 117, 8298-8313.

(38) Fernández, E.; Soler, J.; Garzón, I.; Balbás, L. Trends in the Structure and Bonding of Noble Metal Clusters. Phys. Rev. B 2004, 70, 165403-165414.

(39) Ding, Y.; Mei, Y.; Zhang, J. Z. H.; Tao, F.-M. Efficient Bond Function Basis Set for $\pi$ - $\pi$ Interaction Energies. J. Comput. Chem. 2007, 29, 275-279.

(40) Guan, P.; Mckenzie, D. R. MD Simulations of Ag Film Growth Using the Lennard-Jones Potential. $J$. Phys.: Condens. Matter. 1996, 18, 8753-8762.

(41) Heinz, H.; Vaia, R. A.; Farmer, B. L.; Naik, R. R. Accurate Simulation of Surfaces and Interfaces of Face-Centered Cubic Metals Using 12-6 and 9-6 Lennard-Jones Potentials. J. Phys. Chem. C 2008, 112, 17281-17290.

(42) Winnik, F. M. Photophysics of Preassociated Pyrenes in Aqueous Polymer Solutions and in Other Organized Media. Chem. Rev. 1993, 93, 587-614.

\section{Author information}

javier.montenegro@usc.es; juanr.granja@usc.es

Center for Research in Biological Chemistry and Molecular Materials (CIQUS), Organic Chemistry Department, University of Santiago de Compostela (USC), Santiago de Compostela, Spain.

Notes

The authors declare the following competing financial interests: C.V.-V. and M.A.L-Q. are scientific advisors and stockholders of Nanogap.

\section{Acknowledgment}

This work was supported by the Spanish Ministry of Economy and Competitivity (Mineco) and the ERDF [CTQ2010-15725, CTQ2013-43264-R, MAT2011-28673-C02-01, MAT2012-36754-C02 -01], by the Xunta de Galicia (GRC2006/132, GRC2013/044, EM 2012/117). J.M. received a Ramón y Cajal contract from the Mineco. We also thank the ORFEO-CINCA network and Mineco through CTQ2014-51912-REDC. All calculations were carried out at the Centro de Supercomputación de Galicia (CESGA). 


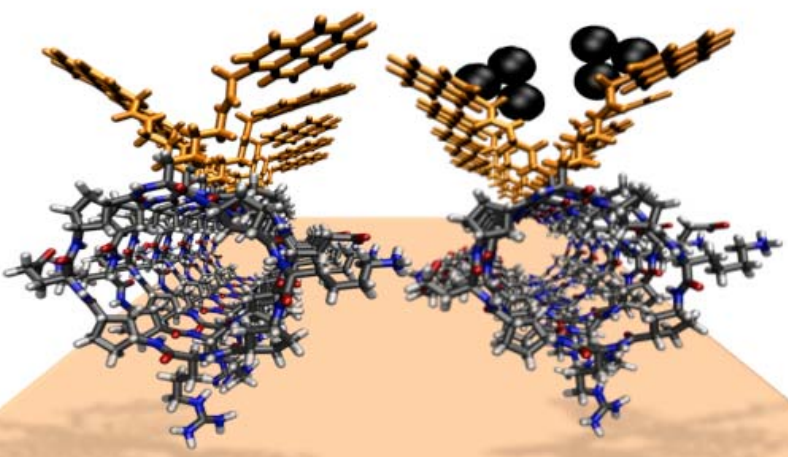

